\title{
A novel approach to track finding in a drift tube chamber
}

\author{
D. Primor, ${ }^{⿴ d d}$ O. Kortner, ${ }^{b}$ G. Mikenberg ${ }^{c d}$ and H. Messer ${ }^{a}$ \\ ${ }^{a}$ The School of Electrical Engineering Tel Aviv University, \\ P.O. Box 39040, Tel Aviv 69978, Israel \\ ${ }^{b}$ Max-Planck-Institut fur Physik, \\ Fohringer Ring 6, D-80805 Munich, Germany \\ ${ }^{c}$ The Weizmann Institute, \\ PO Box 26, Rehovot 76100, Israel \\ ${ }^{d} C E R N$, \\ CH-1211, Geneve 23, Switzerland \\ E-mail: david.primorecern.ch
}

ABSTRACT: Drift tube chambers are commonly used in many experiments in High Energy Physics (HEP). This paper addresses the problem of particle track finding in a drift tube chamber. Although drift tubes have, in general, a high efficiency to detect the passage of particles, in a high radiation background some of the particle hits will be masked by background hits. Under the assumption of high tube efficiency, a novel track finding algorithm, denoted as the Drift Tube Hough Transform (DTHT) algorithm, is presented. The DTHT algorithm uses the possible explanations for a lack of particle hits as additional information, and takes into account all possible scenarios that may occur in the tubes. The DTHT is implemented with a novel extension of the Hough transform and employs a "detect before estimate" approach that first finds the track candidates and then estimates the track parameters. In order to evaluate the performance of the DTHT algorithm, the algorithm was applied to the Monitored Drift Tube (MDT) of the ATLAS experiment and tested using a muon test beam in a high radiation background. It is shown that the use of the additional information reduces the number of fake track rate significantly. A comparison between the DTHT algorithm and the currently best performed program in the ATLAS software, demonstrated that the DTHT algorithm can achieve higher efficiency while reducing the algorithm complexity.

KeYWORDS: Particle tracking detectors; Particle identification methods; Pattern recognition, cluster finding, calibration and fitting methods; Analysis and statistical methods.

\footnotetext{
* Corresponding author.
} 


\section{Contents}

\begin{tabular}{|ll|}
\hline 1. Introduction & 1 \\
\hline
\end{tabular}

\begin{tabular}{|lr}
\hline 2. Tracking in the MDT chamber & 2 \\
\hline
\end{tabular}

\begin{tabular}{|ll|}
\hline 3. The track identification approach & 4 \\
\hline
\end{tabular}

\begin{tabular}{lr|}
\hline 4. The DTHT algorithm & $\mathbf{7}$ \\
\hline 4.1 The Hough transform & 7 \\
\hline 4.2 The track finding phase & 8 \\
\hline 4.3 The line fitting phase & 10 \\
\hline
\end{tabular}

\begin{tabular}{ll|}
\hline 5. Results & 11 \\
\hline
\end{tabular}

\begin{tabular}{ll}
\hline 6. Discussion & 12 \\
\hline
\end{tabular}

\begin{tabular}{lc|}
\hline 7. Conclusions & 14 \\
\hline
\end{tabular}

\section{Introduction}

Particle tracking in High Energy Physics (HEP) experiments is a very demanding task. The Large Hadron Collider (LHC) accelerator [1] and its experiments present new challenges for particle tracking. The high radiation background expected in the LHC environment requires track finding algorithms that handle many background hits and provide high robustness towards noise.

Drift tube chambers [2] are commonly used in many experiments in HEP, providing good hit position measurements. The drift tubes are, in general, very efficient, i.e. a particle that crosses the tube produces a hit with a very high probability. In a high radiation background, the drift tubes suffer from background hits, which often deteriorate the measurements of the particles of interest. In such environment, naïve tracking algorithms (see, for example, the algorithm described by D. Adams et al. [3]) have high fake track rate. An algorithm that takes into account the radiation background effects and hence reduces the fake track rate was developed by the ATLAS collaboration [4].

This paper presents a novel algorithm for track finding in a drift tube chamber. The algorithm, denoted as the Drift Tube Hough Transform (DTHT) algorithm is based on several ideas:

a. Assuming a high efficiency of the drift tubes, the algorithm uses all available information that may explain the cases where a particle crosses the tube but does not produce a hit.

b. The algorithm employs a "detect before estimate" approach that first finds the track candidates and then estimates the track parameters. 

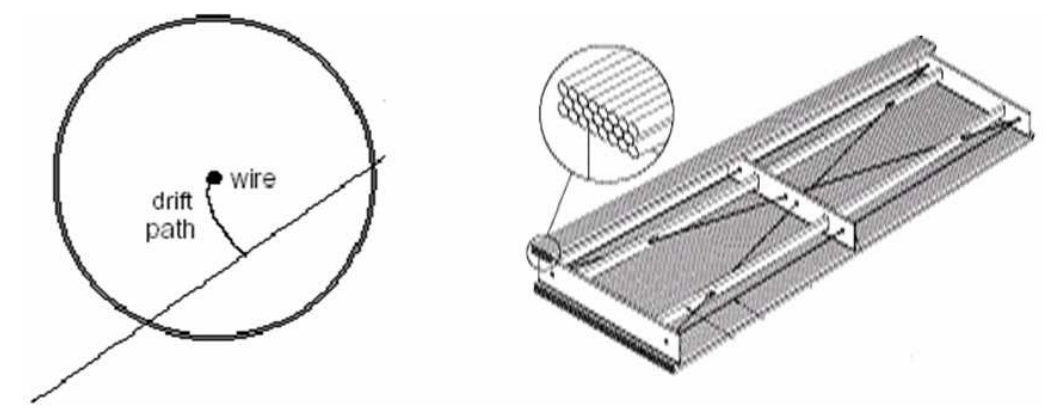

Figure 1. An electron avalanche through the anode wire in a drift tube (left). Schematic drawing of an MDT chamber (right) [1].

c. The algorithm uses a novel modification of the Hough transform for finding the particle tracks.

In order to evaluate the tracking performance of the DTHT algorithm in a high radiation background, the algorithm was implemented on muon tracking through the Monitored Drift Tubes (MDT) chambers of the ATLAS experiment [5] The algorithm was tested using a muon test beam in conjunction with a photon source, and compared to a naïve algorithm [3] as well as to the MuonBoy program [4], which represents the best performing algorithm used within the ATLAS software.

The description of the DTHT algorithm in this paper has been adapted to the muon tracking problem within the MDT chamber. The algorithm is, however, general enough to be used for any other drift tube chamber.

The rest of the paper is organized as follows: in section 2 the MDT chamber and the track finding problem are described. In section 3 the new tracking approach and its geometrical representation to the tracking problem are presented. In section 4 the DTHT algorithm, based on an extension of the Hough transform, is described. Results of test beam data are shown in section 5 , and are discussed in section 6 . The conclusions are presented in section 7

\section{Tracking in the MDT chamber}

The ATLAS Muon Spectrometer [4] is located in a high radiation background environment (mostly neutrons and photons) which makes the muon tracking a very challenging task. The Monitored Drift Tube chambers (MDTs) provide the tracking device in most of the spectrometer area. The basic MDT detection element is a cylindrical aluminum drift tube of $30 \mathrm{~mm}$ diameter and length in a range of 0.9 to $6.2 \mathrm{~m}$. An anode wire of $50 \mu \mathrm{m}$ diameter is positioned along the tube. The tubes are filled with Ar:CO2 (93:7) at an absolute pressure of 3 bars. Operated at a high voltage of $3080 \mathrm{~V}$ corresponding to a gas gain of 20,000 , the tubes have an average spatial resolution of $80 \mu \mathrm{m}$.

An MDT chamber consists of two multi-layers, each formed by three or four layers of tubes. Chamber mechanical deformations are monitored by an optical system; hence the name 'Monitored Drift Tube Chambers'. Muons traversing a drift tube ionize gas molecule along their path. The ionized cluster of electrons drifts towards the anode wire and a charge avalanche develops. The output signal is the digitized drift time which is followed by a dead-time period set to the maximum drift time of about $790 \mathrm{~ns}$. Figure 1 describes the drift path of the ionized cluster to the wire and the MDT chamber geometry.

The particle hit position can be measured using a so called r-t relation. The time it takes the ionized cluster of electrons to reach the anode wire and generate an electric signal is 




Figure 2. Example of a track, given a set of drift circles. One should find all possible lines that are tangent to a minimum subset of drift radii.

proportional to the distance between the particle hit and the wire. Using the $\mathrm{r}$-t relation it is possible to calculate the distance of the hit position to the wire. Given a set of particle hit radii (drift circles), one should find the muon track and estimate its parameters. Since the curvature of the muon track in an MDT chamber is negligible, the local tracking problem is solved by finding all the possible straight lines given a set of hit measurements. Figure 2 describes an example of this tracking problem.

The ATLAS muon chambers experience high neutron and photon background count rates, which have a significant impact on their performance [6],[7], At the LHC design luminosity of $10^{34} \mathrm{~cm}^{-2} \mathrm{~s}^{-1}$, count rates of up to $500 \mathrm{~Hz} \mathrm{~cm}$ (including a safety factor 5) are expected. This corresponds to a count rate of $300 \mathrm{kHz}$ per tube for the longest tubes in the region of high background. The neutron and photon background is caused by the hadrons in the proton-proton collisions that interact with the calorimeters, the shielding material, and the accelerator elements. As the muon chambers are operated in an open geometry environment instead of being between iron absorbers, thermalized neutrons from the hadronic interactions and photons penetrate the muon spectrometer and hit the muon chambers.

In order to test the tracking performance of the MDT chambers at the ATLAS background rates, one of the largest MDT chambers of the ATLAS detector was equipped with final electronics and operated in the Gamma Irradiation Facility (GIF) at CERN in summer 2003 and 2004. This facility provides a $100 \mathrm{GeV}$ muon beam and a $664 \mathrm{GBq}^{137} \mathrm{Cs}$ source emitting $662 \mathrm{keV}$ photons used to simulate the ATLAS background. A detailed description of the GIF and the detector under test can be found in [8]

The neutron and photon background reduces the muon detection efficiency of the tubes. After a hit is detected, the read-out electronics cannot detect another hit within the fixed dead time of $790 \mathrm{~ns}$ after the first hit. Hence, a muon hit is not detected when it falls into the deadtime window after a preceding background hit.

The naïve track reconstruction tries to find all tracks with at least $\mathrm{N}$ muon hits, where $\mathrm{N}$ is a predefined number, smaller or equal to the number of layers in the chamber. A muon hit is defined as a drift radius whose distance from the actual muon track is smaller than 3 times the tube spatial resolution [8], Figure 3 shows the percentage of events with $\mathrm{N}=2,3$, and 4 for the test-beam data sets. The curves in figure 3 correspond to the maximum track finding efficiency which can be achieved if one uses $\mathrm{N}=2,3$, or 4 in the track finding algorithm. It can be seen that 


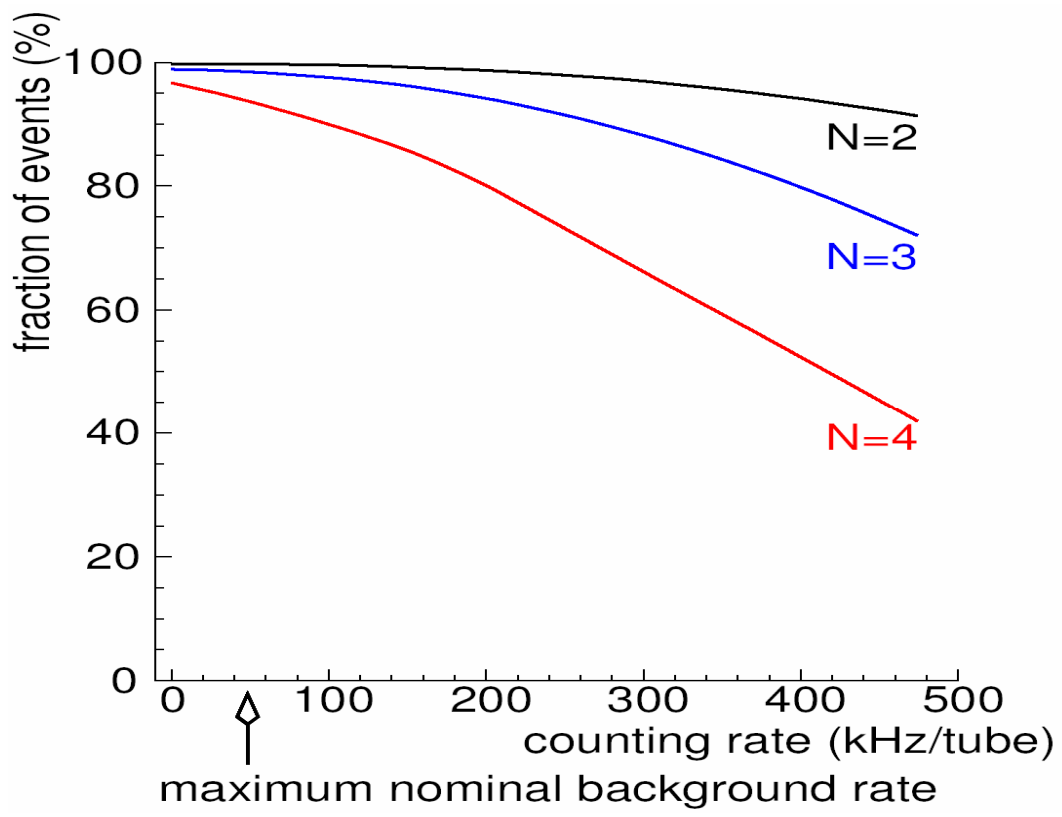

Figure 3. Fraction of muon detected events with at least $\mathrm{N}=2,3,4$ muon hits, as a function of the background rate, measured in the test beam. A muon hit gives a correct drift radius, i.e., the distance between the muon track and the drift radius of a muon hit is smaller than 3 times the tube spatial resolution.

the track finding algorithm have to look for tracks with at least 3 muon hits if a track finding efficiency above $95 \%$ is to be achieved. Unfortunately, the number of fake tracks increases with decreasing $\mathrm{N}$. It is shown in the next sections that the use of additional information can decrease the fake track rate without decreasing the efficiency.

\section{The track identification approach}

The ATLAS drift tubes have full efficiency in their sensitive regions, i.e. whenever a muon traverse a tube, this tube produces an electrical signal (hit). In the presence of photon and neutron background, due to the dead time in the MDT read-out electronics, the drift radius of this hit might not correspond to the distance of the traversing muon from the anode wire of the tube. The proposed approach is to use the high efficiencies of the drift tubes and not only the muon drift radii in order to solve the track finding problem. The suggested approach uses additional information that explains the reason for getting wrong radii or no hit when a muon crosses the tubes. For example, a preceding background particle hit can explain the wrong drift radius output, and a muon that crosses the tube wall can explain the reason of a lack of a hit in one of the chamber layers. Part of this additional information was used in [4],[9],[10] and was proven to be useful for improving the track finding performance. The use of the hits with the correct radii (muon hits), together with the additional information limits the fake track rate; those tracks that are not associated with information that explains the lack of muon hits are rejected.

Since the drift time of the electron clusters in the tube is relatively long compared to the proton beam collision rate, another muon detector (trigger chamber) provides a reference time $t_{0}$. It is the earliest time one may expect the muon to pass the tube. This time together with the maximum possible drift time $(790 \mathrm{~ns})$, define a time window $\left[t_{0}, t_{0}+790\right]$ in which a muon is 


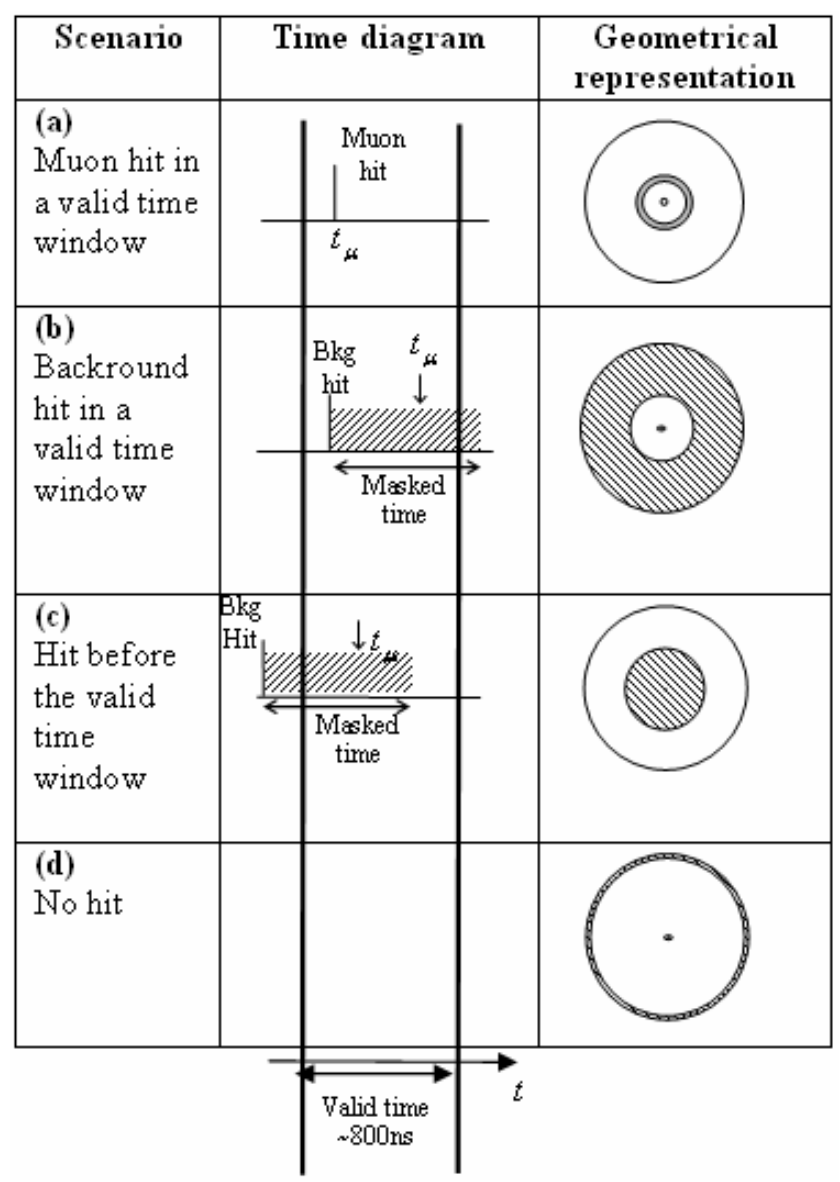

Table 1. The basic scenarios given that the muon crosses the tube. The left column describes the scenario, the middle column describes the hit time, and the right column describes the geometrical representation of the possible muon track regions. The diagonal patterned area in the middle column describes the tube dead time. The grey diagonal patterned area in the right column describes the possible regions in which the muon track may cross the tube. The time $t_{\mu}$ in cases (b) and (c) is an example of a possible muon hit time.

expected to be identified. Since the tube, after it fires, has a dead time of about $790 \mathrm{ns,} \mathrm{a}$ background particle coming within the time window of $\left[t_{0}-790, t_{0}+790\right]$ has a potential to mask the muon. For each tube crossed by the muon, there are several basic scenarios, described in table 1 For each scenario, the possible regions that the muon may cross the tube are drawn.

In scenario (a), a muon particle with hit time within the time window causes the tube to fire. The grey ring represents the distance of the possible tracks to the tube center. Its radius is the measured drift radius, and its width is proportional to the drift radius measurement error. In scenario (b) a background particle with hit time within the time window causes the tube to fire. Since the muon track is masked by the background particle track, it could cross the tube anywhere between the measured background particle radius and the tube wall without getting the tube to fire. This possible distance between the muon track and the tube center is represented geometrically by the grey area within the tube. In scenario (c) a background particle with a hit time preceding the time window causes the tube to fire. Since the muon track can be masked by the background particle track, it can cross the tube anywhere between the tube center and the radius appropriate to the background hit plus the dead time (790 ns). Similarly to the previous 


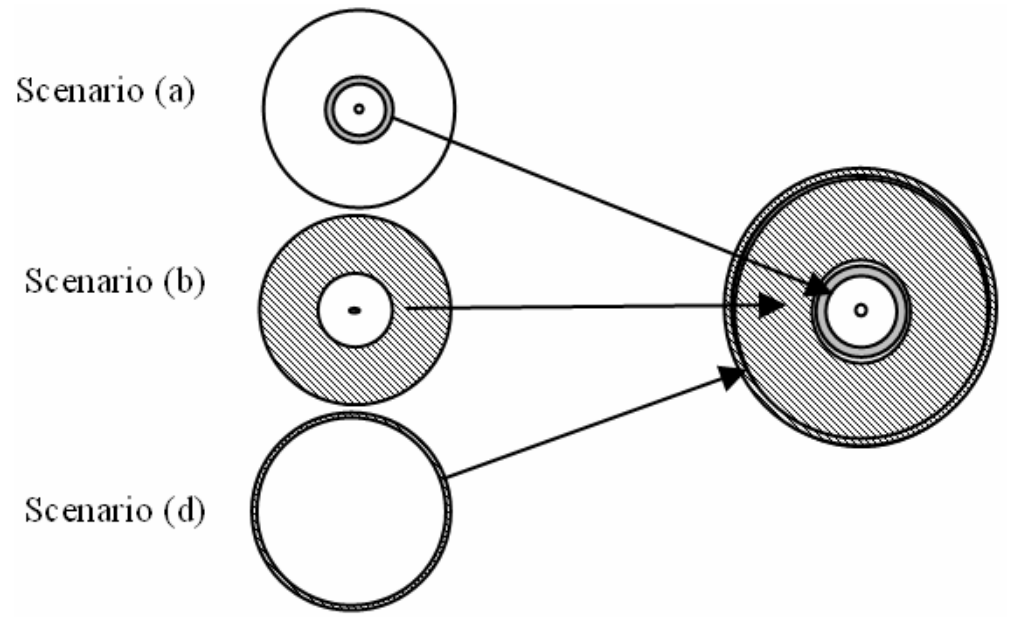

Figure 4. An illustration of all possible regions the muon may cross given a drift radius measurement. The geometrical representation is the combination of three basic scenarios (a), (b) and (d) of table 1.

case, the possible distance between the muon track and the tube center is represented geometrically by the grey area within the tube. In scenario (d) the muon crosses the tube wall, and therefore the tube does not fire. This scenario can also be applied for tubes that are known to malfunction. The possible muon tracks that cross the tube are geometrically represented by a grey ring with radius equal to the tube wall, and width equal to the tube wall width.

Using table 1 it is possible to geometrically represent all the possible regions the muon may cross. This geometrical representation is the combination of the basic scenarios of table 1 . For example, given one measured drift radius, three scenarios are possible; the tube fired due to the passage of the muon (scenario (a)), the tube fired because of the background particle and the muon hit was masked by the background particle (scenario (b)), and the tube fired because of the background particle and the muon hit crossed the tube wall. Thus, the total geometrical representation for this case is the combination of basic scenarios (a), (b) and (d) as described in figure 4.

The track candidate is modeled as a straight line with parameters $(\rho, \varphi)$. Each track candidate crosses the possible muon track regions. The sum of the i-th basic scenarios the track crosses is represented by $H_{i}(\rho, \varphi) \in(0,1, \ldots, M)$ where $M$ is the number of tubes the track crosses. Figure 5 provides an example of the geometric representation of the tracking problem. The function $H_{i}\left(\rho_{0}, \varphi_{0}\right)$ for the line with parameters $\left(\rho_{0}, \varphi_{0}\right)$ in figure 5 is:

$$
H_{i}\left(\rho_{0}, \varphi_{0}\right)=\left\{\begin{array}{lll}
3 & i=1 & \text { scenario }(a) \text { for layers } 1,2,4 \\
1 & i=2 & \text { scenario }(b) \text { for layer } 6 \\
1 & i=3 & \text { scenario }(c) \text { for layer } 3 \\
1 & i=4 & \text { scenario }(d) \text { for layer } 5
\end{array}\right.
$$

A weighted sum of $H_{i}(\rho, \varphi)$ is defined as:

$$
\Lambda(\rho, \varphi)=\sum_{i=1}^{4} H_{i}(\rho, \varphi) \cdot w_{i}
$$




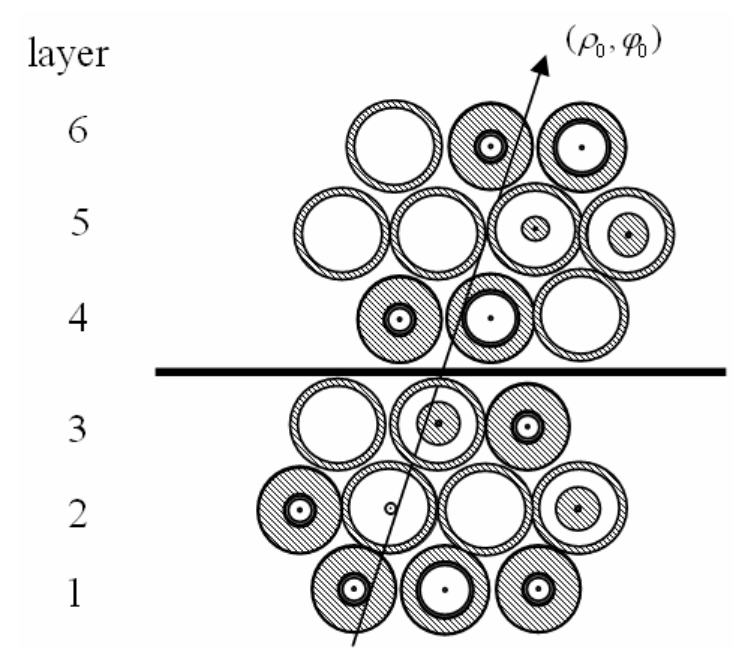

Figure 5. An example of the track finding problem. The geometrical representation for each tube is the combination of the basic scenarios of table 1 . The track candidate with parameters $\left(\rho_{0}, \varphi_{0}\right)$ crosses one possible track region for each tube, corresponding to one scenario.

where $w_{i}=p\left(\theta_{i}\right)$ are the weights for each basic scenario. During the calibration process one can determinate the background level and measure the empirical probability $p\left(\theta_{i}\right)$ for each scenario of table 1 It is assumed that the background level is uniform over a chamber, and thus $p\left(\theta_{i}\right)$ is constant for a chamber. However, since different chambers are placed in different locations, the probabilities $p\left(\theta_{i}\right)$ might not be the same for all chambers.

A muon track is detected if $\Lambda(\rho, \varphi)$ has a local maximum above a predefined threshold $\lambda$ :

$$
\Lambda(\rho, \varphi)>\lambda
$$

In the next section, an algorithm, denoted as the DTHT algorithm, is described. The algorithm, which is based on a modification of the Hough transform, calculates $\Lambda(\rho, \varphi)$ and finds its local maxima above a pre-defined threshold.

\section{The DTHT algorithm}

The proposed DTHT algorithm can be divided into two phases, following the 'detect before estimate' approach. The first phase is used for finding the tracks (track finding), and the second phase is used for estimating the parameters of those tracks (track fitting). The track finding phase is based on a modification of the Hough transform, which numerically solves the problem described in (3). Then, during the track fitting phase, all found tracks are fitted using an iterative procedure.

\subsection{The Hough transform}

The identification of a relatively short track segment within a particle detector can be translated to line identification in a noisy image [11]. The detection of straight line-segments in images is a problem that often occurs in image analysis. One method for detection of collinear points is related to the Hough transform (HT) [12],[13],[14]. Points in the image are transformed into lines in a line parameter space. Lines in the parameter space corresponding to collinear points will cross each other at one point. This point defines the spatial parameters of the line through the collinear points. 


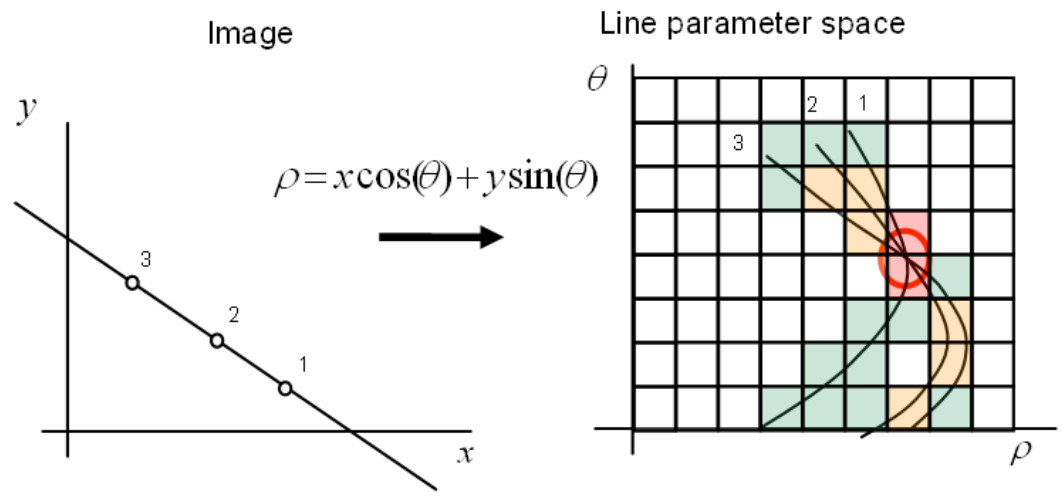

Figure 6. Points in the image (left) are transformed into lines in a line parameter space (right). Lines in the parameter space corresponding to collinear points will cross each other at one point.

In practice, the parameter space is divided into an array of discrete cells. When a point in the image space is transformed into a line in the parameter space, all cells crossed by the line are incremented. If $n$ points are approximately collinear, the line parameters in the image space correspond to a local maximum in the parameter space, produced by the approximate intersection of $n$ lines. Thus, the Hough transform reduces searching for collinear points in the image to looking for cells in the parameter space which are local maxima. Figure 6 illustrates the transform of three collinear points in an image to the Hough parameter space.

An extension of the Hough transform is suggested in [15] where each point $\left\{T_{m} \mid m=1, \ldots, M\right\}$ has a circularly symmetric scalar cost field $C_{m}(r)$. The cost of a path $l$ is increased by $C_{m}(r)$ if the Euclidean distance from the path to the point $T_{m}$ is $r$. The optimal path is the one whose cost is minimal. This optimal path is found by a Hough-like parameter space approach, in which an extended Hough transform function $C(\rho, \theta)$ represents for a pair of normal parameters $(\rho, \theta)$ the cost of respective path.

\subsection{The track finding phase}

The DTHT track finding phase is based on an extension of [15] and uses a novel modification of the Hough transform. The basic scenarios described in table 1 are used as different layers in the Hough parameter space $\mathbf{H}(\rho, \varphi)$ :

$$
\mathbf{H}(\rho, \varphi)=\left[H_{1}(\rho, \varphi), H_{2}(\rho, \varphi), H_{3}(\rho, \varphi), H_{4}(\rho, \varphi)\right]
$$

For each tube, the possible muon track regions described in section 3 are used for the transform. For a pair of normal parameters $(\rho, \varphi)$, the Hough transform function $H_{i}(\rho, \varphi)$ represents the number of tube possible regions, associated with the basic scenario $i$, that were crossed by the respective track. The layer $i$ of the parameter space $H_{i}(\rho, \varphi)$ is represented by a set of $N_{\rho} \cdot N_{\varphi}$ accumulators or cells in the $(\rho, \varphi)$ space, where $N_{\rho}$ and $N_{\varphi}$ are the number of sample points in the $(\rho, \varphi)$ space.

Let $\left\{T_{m} \mid m=1, \cdots, M\right\}$ be a set of $M$ tubes centers inside a MDT station. Let $L$ be the set of straight lines that traverse the station. Every tube center $T_{m}$ is characterized by its polar coordinates $\left(\rho_{m}, \varphi_{m}\right), 0 \leq \rho_{m}<\rho_{\max }, 0 \leq \varphi_{m}<2 \pi$. A track $l$ is represented by its normal 


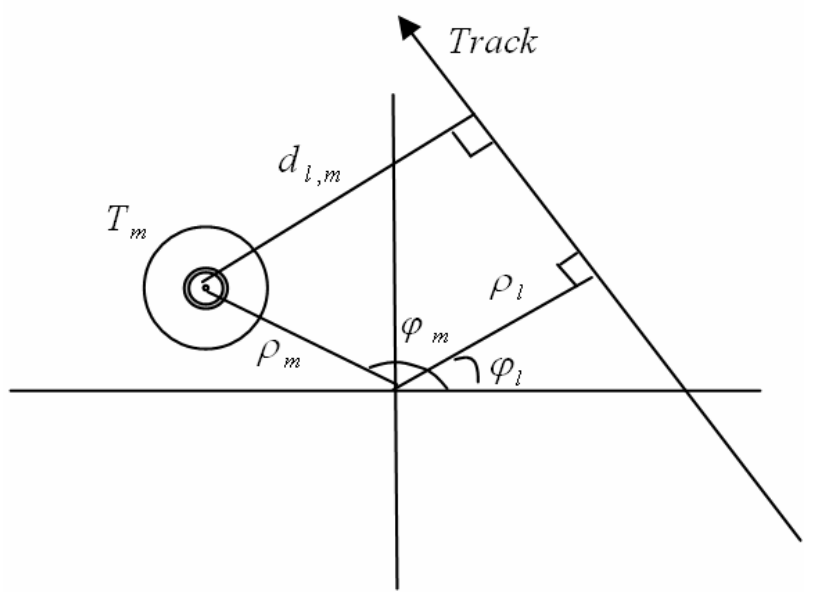

Figure 7. Tube center and track representation.

The tube space

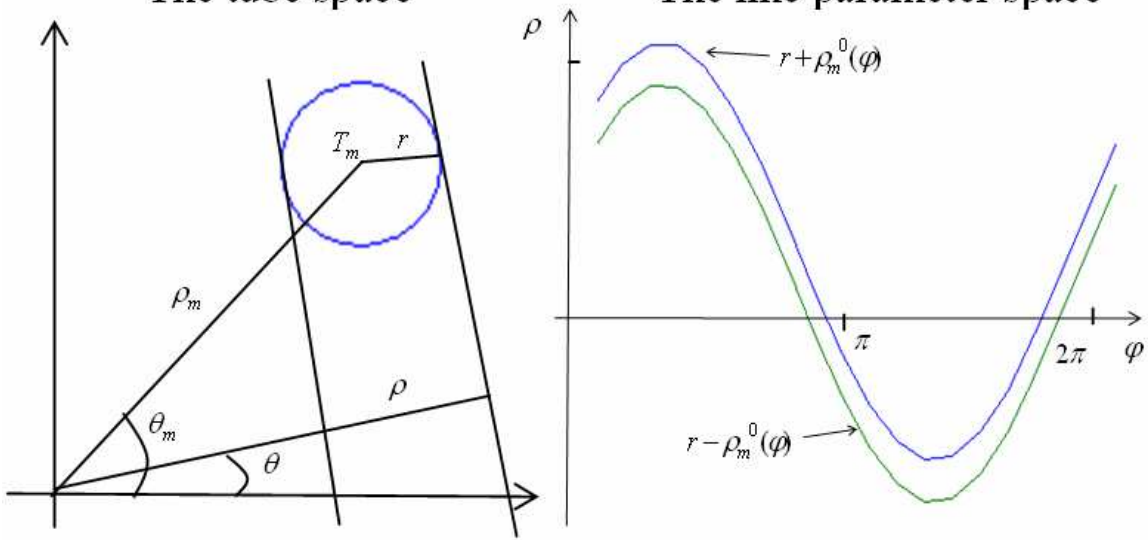

Figure 8. Typical lines at distance $r$ from $T_{m}$ (left) and the pair of sinusoids in the parameter space (right).

parameters $\left(\rho_{l}, \varphi_{l}\right), 0 \leq \rho_{l}<\rho_{\max }, 0 \leq \varphi_{l}<2 \pi$. The origin of a polar coordinate system is located at the center of the chamber, as shown in figure 7.

Around each tube $T_{m}$ there exists a set of possibly 4 muon track regions; each corresponded to a different basic scenario, as described in table 1. Each muon track region is digitized, providing a set of discrete radii. For each tube $T_{m}$ and for each discrete radius $r$, a set of tangent straight line parameters are calculated (figure 8) according to:

$$
\begin{aligned}
& \rho=r+\rho_{m}{ }^{0}(\varphi)=r+\rho_{m} \cos \left(\varphi_{m}-\varphi\right), \varphi \in[0,2 \pi) \\
& \rho=-r+\rho_{m}{ }^{0}(\varphi)=-r+\rho_{m} \cos \left(\varphi_{m}-\varphi\right), \varphi \in[0,2 \pi)
\end{aligned}
$$

or

$$
\left|\rho-\rho_{m}{ }^{0}(\varphi)\right|=r
$$

The solution $(\rho, \varphi)$ of (5) is quantized, and the appropriate accumulator in the Hough space is incremented. At the end of the accumulation phase, each Hough space layer $H_{i}(\rho, \varphi)$ holds the sum of tubes with basic scenario $i$ that were crossed by the track with normal parameters $(\rho, \varphi)$. 
Let $W(\rho, \varphi)$ be a function of the multi-layers Hough space:

$$
W(\rho, \varphi)=f(\mathbf{H}(\rho, \varphi))
$$

where the function $f()$ defines the approach used.

In the search phase $W(\rho, \varphi)$ is searched for a local maximum above a predefined threshold. For example,

$$
W(\rho, \varphi) \equiv H_{1}(\rho, \varphi)
$$

is related to the case where no other information but the drift radii are used, as in figure 2 However, if different weights are given to the different basic scenarios, then one can define:

$$
W(\rho, \varphi) \equiv \sum_{i=1}^{4} H_{i}(\rho, \varphi) \cdot p\left(\theta_{i}\right)
$$

and thus:

$$
W(\rho, \varphi)=\Lambda(\rho, \varphi)
$$

where $\Lambda(\rho, \varphi)$ is defined in (2) with $w_{i}=p\left(\theta_{i}\right)$.

In order to calculate an approximated solution to (3), one should find local maxima of $W(\rho, \varphi)$ that are above a pre-defined threshold $\lambda$ :

$$
\max _{\rho, \varphi} W(\rho, \varphi)>\lambda
$$

While in (3) the problem is described for continuous values of the line parameters $(\rho, \varphi)$, in (10) the parameter space is sampled into a set of discrete values, and the maximization problem is solved numerically for discrete set of line parameters. The size of the parameter space determines the complexity of the Hough algorithm in one hand and the accuracy in the other hand. The calculation of (10) can be done using different real time techniques for two dimensional search described e.g. in [12]

\subsection{The line fitting phase}

In order to get accurate track parameter estimation, line fitting is applied to the muon track candidates. It is assumed that the track is a straight line in the chamber, and that the drift radius error is Gaussian. Although the second assumption is only an approximation (especially for small radii), it was shown in [10] that non-Gaussian errors have a small impact on the track fitting performance. Following the iterative fitting technique suggested in [16], the initial values of the track parameters are the results of the Multi-layers Hough transform algorithm. This algorithm identifies the hits which were produced by the muon (muon hits) and those which were produced by the background particles that masked the muons. Then, the hit radii to be fitted are those which were identified as muon hits. The track parameters are estimated by fitting the track to the drift circle radii. This is done by minimizing the $\chi^{2}$, which is given by:

$$
\chi^{2}=\sum_{i=1}^{n} \frac{\left(\Delta_{i}-r_{i}\right)^{2}}{\sigma_{i}^{2}}
$$

where $\Delta_{i}$ is the distance from the track to the wire and $\sigma_{i}^{2}$ is the variance at the measurement drift radius $r_{i}$. In a high radiation background, where the number of track candidates is increased, it is possible to use the $\chi^{2}$ value as another criterion to reject fake tracks, i.e. track candidates with a $\chi^{2}$ value higher than a certain threshold are excluded. 


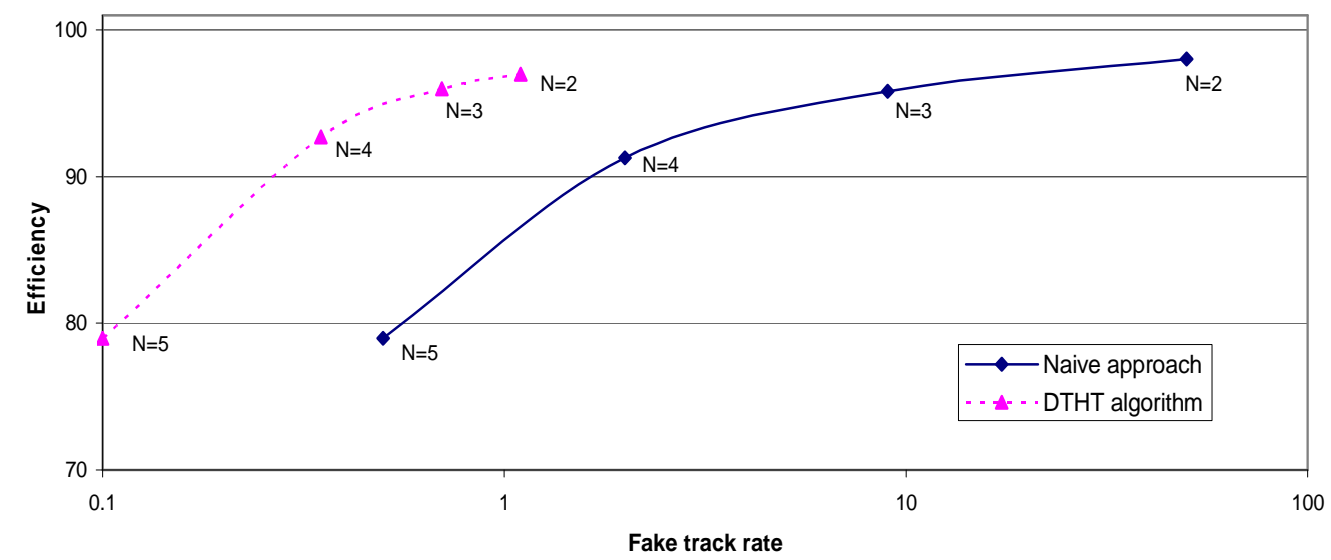

Figure 9. Number of fake tracks vs. track finding efficiency, for the naïve approach (solid line) and for the DTHT algorithm (dashed line). The points on the curves are the minimal number of collinear hits used in the algorithm $(\mathrm{N}=2,3,4,5)$.

\section{Results}

The DTHT algorithm has been applied to the data of test beam for background rate varying from $0 \mathrm{kHz}$ per tube (no noise) till $400 \mathrm{kHz}$ per tube (about 7 times the nominal ATLAS background level). Although all the tubes in the chamber suffer from the high radiation, only those tubes whose distance to the real track is less than $150 \mathrm{~mm}$ were used for track finding. This is similar to the ATLAS environment, where a trigger chamber defines a road in which a muon can be identified. The track reconstruction efficiency is defined as the ratio $n_{M D T}^{(3 \sigma)} / n_{\text {ext }}$, where $n_{\text {ext }}$ denotes the number of tracks reconstructed by the precision external silicon tracker ("truth") and $n_{M D T}^{(3 \sigma)}$ is the number of tracks reconstructed in the muon chamber, whose distance to the "truth" is smaller than 3 time the chamber resolution. The chamber resolution is the standard deviation error of the estimated muon tracks position for clean events (without a radiation background) [8]. The fake track rate is the average number of false tracks reconstructed per real muon. It is defined as $\left(n_{M D T} / n_{e x t}\right)-1$, where $n_{M D T}$ is the total number of tracks reconstructed by the algorithm assuming that in each event there is one real muon track.

Figure 9 depicts the fake track rate vs. the track finding efficiency for the naïve algorithm calculated using (7), and for the DTHT algorithm, calculated using (8), for background level of $80 \mathrm{kHz}$ per tube. The naïve algorithm (similar to [3],[8]] tries to find at least $\mathrm{N}(\mathrm{N}=2,3,4,5)$ collinear hits without using any additional information, whereas the DTHT algorithm uses that information for improving the track detection performance.

Both the naïve algorithm and the DTHT algorithm can reach 97\% efficiency. However, the naïve algorithm suffers from high fake track rate, especially for an efficiency higher than $95 \%$, whereas the fake track rate for the new algorithm is negligible. It can be seen from figure 9 that the use of the DTHT algorithm reduces the fake track rate significantly, without reducing the probability of track detection. 


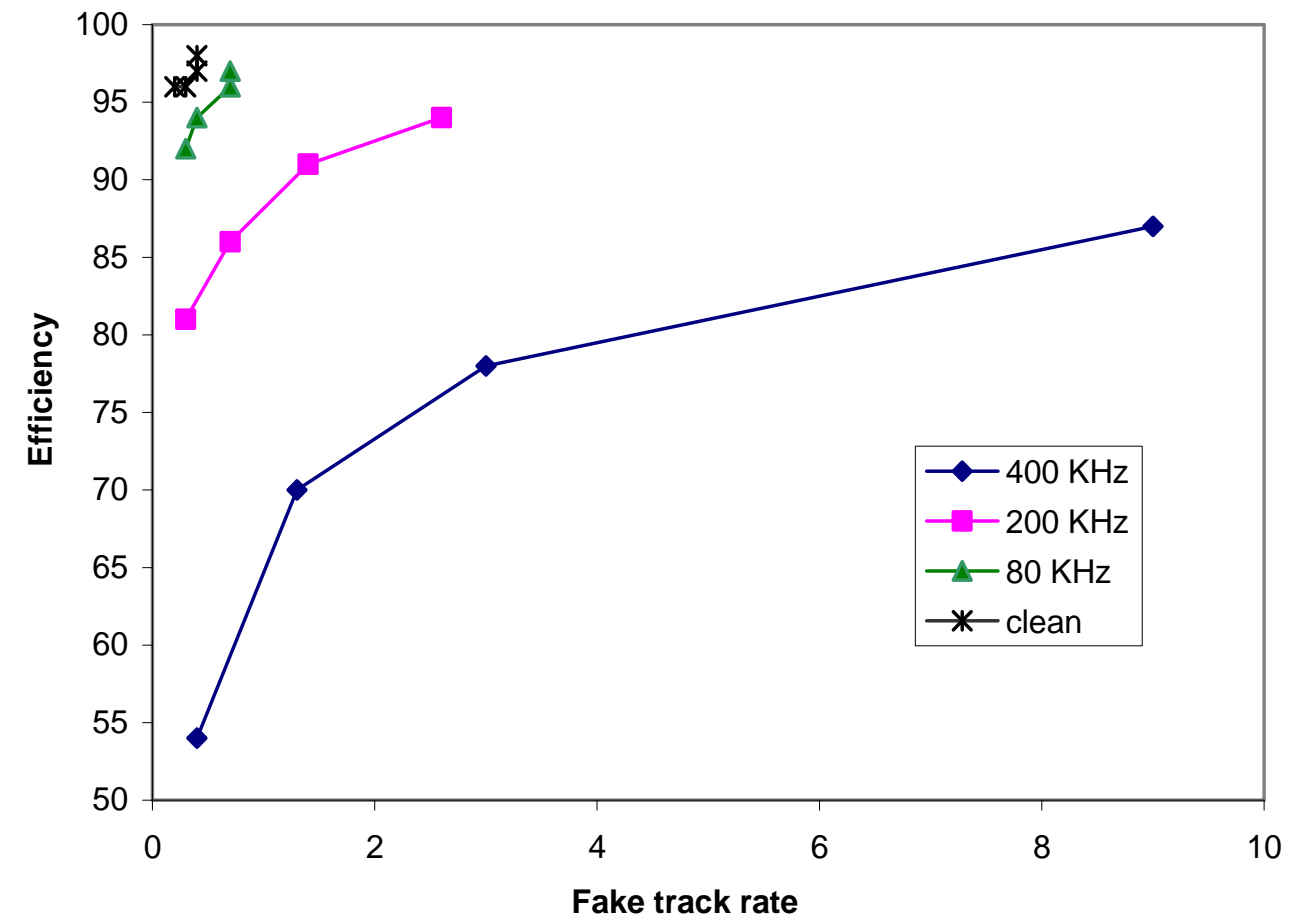

Figure 10. Fake track rate vs. track finding efficiency of the DTHT algorithm. The different lines are the optional tradeoff between fake rate and efficiency of the algorithm for different background levels.

Figure 10 depicts the track finding efficiency as a function of the fake track rate achieved by the DTHT algorithm for different background rates. Four lines are shown in figure 10 The lower line corresponds to the higher background rate, whereas the higher corresponds to the clean case (no radiation background). Each line consists of 4 points, each for different set of parameters, $\chi^{2}$ and $\lambda$. It is possible to adjust the efficiency and fake rate values by changing the $\chi^{2}$ threshold of (11) and the Hough threshold $\lambda$ of (10). It can be seen that it is possible to achieve efficiencies higher than $93 \%$ with reasonable fake track rates for background radiation rate lower than $200 \mathrm{KHz} /$ tube. For background radiation rate higher than $200 \mathrm{KHz} /$ tube, the fake track rate increases significantly. Note that for these very high background rates, about half of the tubes are masked by background particles.

\section{Discussion}

The DTHT algorithm uses more information than the naïve algorithm, and thus it significantly reduces the fake track rate while keeping the efficiency at the same level, as shown in figure 9. The DTHT algorithm was also compared to the one used in MuonBoy [4], which is the currently leading algorithm for muon reconstruction within the muon spectrometer in ATLAS. The Muonboy algorithm is based on combinatorial search and uses a non-symmetric penalty factor in its line fit calculation in order to suppress background hits in the track fit. While in the DTHT algorithm all the information is processed in a one Multi-Layers Hough transform, the MuonBoy heuristic algorithm assigns penalty to many segment combinations, and makes a heuristic decision based on the segment penalty factor. 


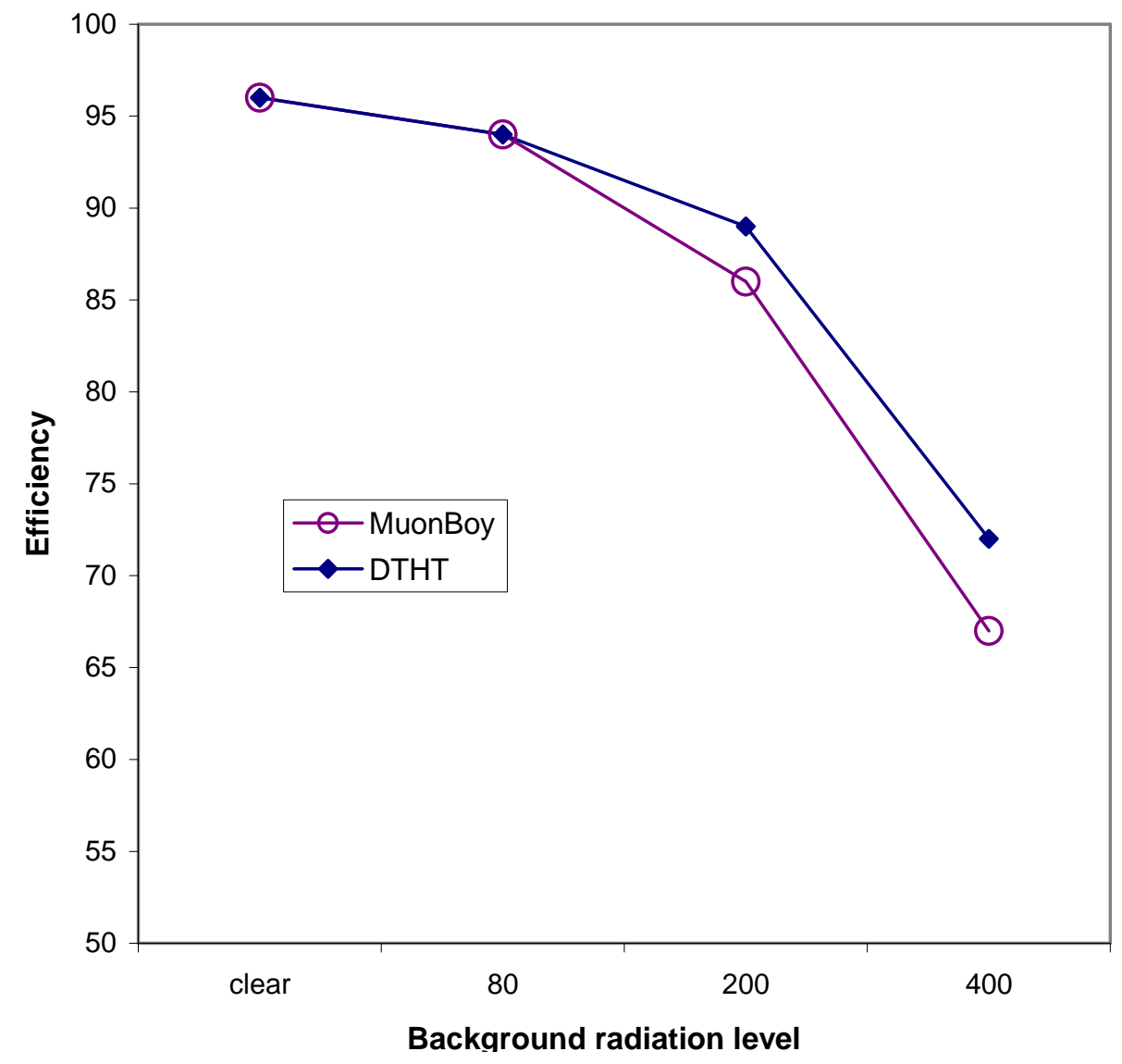

Figure 11. Track finding efficiency vs. the background radiation rate of the DTHT and the MuonBoy algorithms. The efficiencies of both algorithms are reduced significantly as the background radiation level increases. The efficiency of the DTHT algorithm is slightly better than the MuonBoy algorithm for high radiation background.

Figure 11 depicts the efficiency as a function of the different background rates for the Muonboy and the DTHT algorithms. The efficiency values taken for the DTHT algorithm are those with approximately the same fake track rate as the MuonBoy algorithm. For both algorithms the efficiency decreases where the background level increases. It can be seen that the performance of the DTHT algorithm is slightly better than the MuonBoy algorithm for a high radiation background.

As demonstrated in figure 10, it is possible to adjust the efficiency and fake rate values of the DTHT algorithm by changing the $\chi^{2}$ and $\lambda$ parameters and thus get a better efficiency while increasing the fake track rate. As for the MuonBoy algorithm, however, higher efficiencies could not be achieved, even for higher fake track rate.

A comparison of the algorithm computational loads can be done separately for the two algorithm phases - the track finding and the track fitting.

During the track finding phase of the DTHT algorithm the Hough transform requires $O\left(M \cdot N_{x_{1}}\right)$ operations for calculating the transform and incrementing the appropriate Hough cell values, and $O\left(N_{x_{1}} \cdot N_{x_{2}}\right)$ operations for the maxima search in the Hough space, where 
$N_{x_{1}}, N_{x_{2}}$ are the dimensions of the Hough space [17] and $M$ is the number of hit points. A combinatorial line search, such as muon boy, requires $O\left(M^{2}\right)$ operations. If $M$ is large it requires significantly more computational load than the Hough transform. In ATLAS, however, the number of the MDT tubes is relatively small, and therefore the number of hits, even in high radiation background, is not very large. Typical values of $M$ can be between 30 and 300, depending on the chamber location. Since the dimensions of the Hough space $N_{x_{1}}, N_{x_{2}}$ are in the same range, both algorithms require about the same number of operations for the track finding phase.

As for the track fitting phase, where the Muonboy algorithm calculates a fit for many line combinations, the DTHT algorithm calculates a fit only for the tracks which where found during the track finding phase. Since the line fitting process is a non-linear iterative procedure that requires many operations, the DTHT algorithm requires fewer operations.

\section{Conclusions}

High radiation environment makes the performance of a naïve tracking method insufficient. A better understanding of the detector can be used to improve the muon tracking performance. Using additional information, the DTHT algorithm significantly reduces the fake track rate.

A comparison with the presently best performing algorithm in the ATLAS muon spectrometer (MuonBoy) shows similar achievable efficiency for a given fake rate. However, the DTHT enables one to easily increase the efficiency while adjusting the fake track rate, where for the MuonBoy algorithm this procedure is not trivial, if at all possible. Moreover, in a high radiation background, the DTHT algorithm requires less computational load.

The DTHT is a general algorithm for track finding in a drift tube chamber. An additional reduction in complexity can be achieved for chambers containing large number of tubes. For such chambers, the Hough transform technique used by the DTHT algorithm reduces the computational load significantly.

\section{Acknowledgments}

We would like to thank Ahmimed Ouraou and Jean-Francois Laporte from the Saclay group for helping us converting the test beam data to a format MuonBoy can read, and running the MuonBoy algorithm for a comparison. We also like to thank Niles Van Eldik that provided us an MDT event viewer and contribute to our MDT understanding.

\section{References}

[1] LHC study group, The LHC conceptual design report, CERN-AC-95-05, 1995.

[2] W. Blum and G. Rolandi, Particle detection with drift chambers, Springer-Verlag (1994).

[3] D. Adams et al., Track reconstruction in the ATLAS Muon spectrometer with MOORE, ATL-SOFT2003-007 [ATL-COM-MUON-2003-012].

[4] Atlas Muon spectrometer Technical Design Report, CERN-LHCC-97-22, 1997. 
[5] ATLAS Collaboration, ATLAS detector and Physics Technical Design Report, CERN/LHCC/99-14, ATLAS TDR 14, 1999.

[6] M. Aleksa et al., Rate effects in high-resolution drift chambers, Nucl. Instrum. Meth. A 446 (2000) 435 .

[7] M. Deile et al., Performance of the ATLAS precision muon chambers under LHC operating conditions, Nucl. Instrum. Meth. A 518 (2004) 65.

[8] S. Horvat et al., Operation of the ATLAS Muon drift-tube chambers at high background rates and in magnetic fields, EEEE Trans. Nucl. Sci. 53 (2006) 562.

[9] J.F. Laporte et al., On the number of layers per multilayer in MDT chambers part I, CERN, 21 Aug. 1996 [ATL-MUON-96-126], [ATL-M-PN-126].

[10] M. Deile et al., Optimization and calibration of the drift-tube chambers for the ATLAS muon spectrometer, CERN-THESIS-2003-016, CERN 2003.

[11] R.Fruhwirth et al., Data analysis techniques for high-energy physics, Cambridge monographs on particle physics, nuclear physics and cosmology, 2000.

[12] J. Illingworth, J. Kittler, A survey of the Hough transform, Comp. Vis. Graph. Im. Proc. 44 (1988) 87.

[13] R.O Duda, P.E. Hart, Use of the Hough transform to detect lines and curves in pictures, Commun. Ass. Comput. Mach. 15 (1972) 11.

[14] P.V.C. Hough, Method and means for recognizing complex patterns, U.S Patent 3,069,654. Dec, 1962.

[15] N. Kiryati and A.M. Bruckstein, On navigating between friends and foes, EEEE Trans. Pattern Anal. Machine Intell. 13 (1991) 602.

[16] J.M. Woudstra, Precision of the ATLAS muon spectrometer, PhD thesis, NIKEF 2002.

[17] N. Kiryati and A.M Bruckstein, What's in a set of points? (straight line fitting), EEEE Trans. Pattern Anal. Mach. Intell. 14 (1992) 496. 